\title{
Influential Article Review - Is There a Migratory Effect? On Opinions Regarding Government Accountability, Russian Migrant Communities in Western Europe Are Compared to Russians in Three Former Soviet Nations
}

\author{
Zayne Pena
}

Aron Murillo

Aniyah Horner

This paper examines migration. We present insights from a highly influential paper. Here are the highlights from this paper: In a number of recent articles migration has been used as a 'natural experiment', which can give insights into the general mechanisms of attitude formation. Studies from the field of psychology, however, suggest that the process of acculturation might be affected by migrants being in a particular situation. If such a 'migration effect' exists, then the conclusions on the more general mechanisms of attitude formation based on migration studies have to be rethought. We test this using a novel study design comparing acculturation in which we compare attitudes in the Russian diaspora left behind, in Estonia, Latvia and the Ukraine, by the collapse of the Soviet Union to those of Russian migrants in Germany, Denmark and the Netherlands. The results show that there are no differences in acculturation between the diaspora in the East and the migrants in the West and therefore no 'migration effect' on acculturation. For our overseas readers, we then present the insights from this paper in Spanish, French, Portuguese, and German.

Keywords: acculturation, natural experiment, migrants, diaspora, Soviet Union

\section{SUMMARY}

- We start by showing the different attitudes of diaspora of Russians living in Estonia, Latvia and Ukraine, Russian migrants living in Denmark, the Netherlands and Germany, natives from Estonia, Latvia and Ukraine, and natives from Denmark, the Netherlands and Germany.

- Figure 2 shows an overall tendency for the migrants and the diaspora to be more in favour in government responsibility than the natives, but also more in agreement with their respective paring of natives in the East and the West. Examining the support for the government providing a reasonable standard of living for the sick, the diaspora and migrants express slightly more support for this than the natives in the Baltics and Western countries. However, considering that the question runs on a scale from 1 to 4 these differences between the groups are very small and, in most cases, insignificant. 
- Regarding government's responsibility to provide a standard of living for the old, we see that the diaspora has the strongest preference for more responsibility. This is followed by the migrants and natives in the East and the West. There is thus a sort of paring where the migrants and the natives in both the East and the West are more internally alike than different.

- The diaspora variable shows the effect of being a part of the diaspora as opposed to being a migrant. A significant effect on the diaspora variable would mean that the Russian diaspora in the Ukraine and the two Baltic countries are different in attitude from Russian migrants in Denmark, Germany and the Netherlands. A negative effect means that diaspora is less in favour of government responsibility than migrants, and a positive sign means the opposite. It appears that there are no significant differences between diaspora and migrants on all the four attitudes, meaning that after controlling for compositional characteristics, there is no difference between the diaspora and migrants in how they adjust to the receiving society. The idea that those who migrate voluntarily have a mind-set or personality which should result in quicker acculturation to the host society, in other words the 'migrant effect', cannot be confirmed.

- Looking at the other effects in Table 2, we can see that natives' attitude has a positive effect on the attitude towards government responsibility for the old, the unemployed, and redistribution. This shows that natives' attitudes affect the attitudes of the Russian diaspora and migrants, except towards responsibility for the sick.

\section{HIGHLY INFLUENTIAL ARTICLE}

We used the following article as a basis of our evaluation:

Hedegaard, T. F., \& Bekhuis, H. (2018). A migration effect? Comparing the acculturation of Russian migrant populations in Western Europe to Russians in three former soviet countries on attitudes towards government responsibility. Comparative Migration Studies, 6(1).

This is the link to the publisher's website: https://comparativemigrationstudies.springeropen.com/articles/10.1186/s40878-018-0072-8

\section{INTRODUCTION}

Large-scale cultural or institutional changes are rare. Though this is good for the stability of society, it also means that it is difficult to test many of the existing theories on how culture and institutions impact attitudes. Except for the few natural experiments, like the East-West German reunification (Svallfors, 2010), there are few opportunities to study instances in which we can follow how attitudes adapt to a new context or cultural changes. Partly for this reason the literature on whether migrants' attitudes acculturate has grown quite substantially over the last several years as, in addition to giving insights into the integration of migrants into new host societies, these studies also help to explore the more general mechanisms of attitude formation. Studies of migrants' attitudes have, in some instances, even been described as 'natural experiments', as they show what happens when a large group of people with varying backgrounds come into contact with a new country having a different culture and different institutions. Though the results of these studies are mixed, the majority do show that migrants acculturate, meaning that their attitudes become more like those held by the native population than those dominant in the country of origin (Breidahl \& Larsen, 2016; Chauvet, Gubert, \& Mesplé-Somps, 2016; Dinesen, 2012; Luttmer \& Singhal, 2008; Maxwell, 2010; Reeskens \& Van Oorschot, 2015; Röder \& Mühlau, 2012; Röder \& Mühlau, 2014; Safi, 2010). This would indicate that attitudes are context-specific and thus changeable.

There might, however, be an issue with this approach of testing the mechanisms of attitude formation. A number of studies, mainly from the field of psychology, have argued that the act of migrating might be affected the willingness to adapt to the values and attitudes in a new country (Berry, Kim, Minde, \& Mok, 1987; Berry, 1997, 2001; Birman \& Trickett, 2001; Boneva \& Frieze, 2001; Main, 2014; Polek, Van 
Oudenhoven, \& Berge, 2011). If this is the case, then conclusions about the more general mechanisms of attitude formation might have to be reconsidered. Therefore, we want to demonstrate whether the 'migration effect' on the ability to acculturate exists.

There are, however, a number of problems related to comparing the impact of the migration dimension on acculturation, as this often entails comparisons of culturally very different groups. To overcome the problems in the literature we outline a novel design in which the collapse of the Soviet Union serves as a natural experiment. The fall of the Berlin Wall in 1989 signalled the beginning of the end for the Soviet Union, and about 2 years later the collapse was final. This meant, among many other things, that the states in the union now became sovereign nations again, which resulted in 25 million Russians becoming a 'part of a large diaspora, without moving an inch or leaving their homes' (Heleniak, 2004). Large communities of Russians therefore live in former Soviet states, and these diasporas make up a sizeable part of the population in countries like Estonia (24.8\%), Latvia (26.2\%), and Ukraine (17.3\%) (CIA World Factbook, 2011). By comparing to what degree the attitudes of ethnic Russian-living the aforementioned countries and Russian migrants living in Western Europe are similar to those of the natives in their respective countries, we will study whether this 'migrant effect' exists.

We will measure the level of acculturation to the attitudes of the natives by combining the 'European Social Survey' (ESS) from 2008 with the 'Migrants' Welfare State Attitudes' (MIFARE) survey from 2016, which was collected among ten migrant groups, including Russians, in the Netherlands, Denmark and Germany. Using this combination we will study attitudes of displaced Russians concerning whether providing healthcare for the sick, a guaranteed standard of living for the old and the unemployed, and resource redistribution should be the responsibility of government.

\section{CONCLUSION}

A number of studies that have come out in recent years focus on migrants' attitudes and whether migrants acculturate, meaning whether they adapt to become more like the natives. The studies predominantly show that migrants' attitudes are context-specific and therefore adaptable (Breidahl \& Larsen, 2016; Luttmer \& Singhal, 2008; Maxwell, 2010; Reeskens \& Van Oorschot, 2015; Röder \& Mühlau, 2014; Safi, 2010). In addition to informing the debate on integration of migrants, these studies have also been used to explore the general mechanisms of attitude formation, as migration is seen as a 'natural experiment' in which we can observe whether changing context can also change attitudes. Drawing on theories, mainly from psychology, we argue that this praxis of using migration to test the mechanisms of attitude formation might be problematic if being a migrant alone has an impact on acculturation.

To explore this we outline a study design relying on a true natural experiment: the collapse of the Soviet Union. This historic event resulted in a diaspora of upwards of 25 million people 'left behind' in countries that used to belong to the union. The Russian diaspora became a minority in the new countries involuntarily. By comparing their acculturation to that of Russians who migrated voluntarily, we are the first to test the 'migration effect'. Compared to the existing literature, this design has the advantage that we can pinpoint the effect of migrating, while keeping the effect of origin country and culture constant. Based on the literature we outlined three possible scenarios: First, that the diaspora acculturates more successfully than the migrants, as predicted by the literature following Berry et al. (1987). Secondly, that the Russian migrants acculturate better than the diaspora, as for instance predicted by the literature on the 'migrant optimism'. Third, that migrants and the diaspora acculturate to the attitudes of the natives about equally well. If we did not find the latter scenario to be the most realistic then current research on the adaptation of attitude formation to a new context might have overestimated, or underestimated, the effect of context and institutions.

To test this we merged the fourth version of the European Social Survey with the Migrants' Welfare State Attitudes survey. By combining the two surveys we were able to compare diaspora Russians in Estonia, Latvia and Ukraine, and Russian migrants in Denmark, the Netherlands and Germany, with each other and with natives in the respective countries. The results showed that the Russian diaspora in the two Baltic countries and Ukraine did in fact prefer a more extensive responsibility for the government in 
providing welfare, which could be interpreted as initial support for the migration effect. However, the results also show that this difference is mainly due to the diaspora on average being older and poorer. Therefore, when controlling for these factors, there was no difference in acculturation to natives' attitudes between migrants and the diaspora.

Although we argue that the 'migrant effect' did not exist, there are some limitations on the research design which should be improved before stating that a migrant personality, regardless of context and

country of origin, does not exist. The perfect design would require the migrants and diaspora to be within the same context. It is, however, hard to imagine a situation in the real world where this could be the case. With that in mind, it is a limitation that we might not capture all contextual differences in the study. Second, we only explore this using Russians as the case. We see no reason why Russians should be more or less likely than other nationalities to acculturate, but more variation in nationality would of course strengthen the conclusion. Third, the Russian diaspora is quite homogeneous in terms of factors like age, income, and education. It would have been good to have more variation to test the impact of these factors; however, given the nature of the experiment this was not possible. Finally, there is the ever-present question of selfselection. According to Heleniak (2004), there has always been a steady flow of migration between Russia and the bordering countries. However, from the 1930s onwards, policies were implemented that facilitated increased migration from Russia to the other states in the union. This resulted in an increased net migration especially to urban areas, in which the Russians could form communities. Though the initial migration was voluntary, it cannot be ruled out that the decision to migrate before the fall of the Soviet Union and not to return after, which 2.1 million Russians did, does signify some level of self-selection. Of the migrants we looked at in Western Europe, less than 1\% migrated before the fall of the union. Therefore, though we don't know specific reasons for migrating, we can presume that the action was voluntary and not a flight from Soviet rule. However, as with those who initially migrated to the Eastern countries but from the opposite point of view, it is impossible to know whether there is an element of self-perceived compulsion.

Despite these limitations, we believe that this study is the best possible test of the 'migrant effect'. For the growing field of migration studies this means that migration can be used to study attitude formation in a more general sense. Migrant surveys show not only attitudes of a select group of people from a certain origin country, but the attitudes of people from a certain origin country who are adapting to their new environment. The question for further research is how fast this process of acculturation of attitudes will go, and which factors affect the speed of the acculturation process.

\section{REFERENCES}

Aasland, A. (1994). The Russian population in Latvia: An integrated minority? The Journal of Communist Studies and Transition Politics, 10(2), 233-260.

Bekhuis, H., \& Hedegaard, T.F. (2016). MIFARE survey: Migrants' welfare state attitudes methodological report. (methodological report). Nijmegen: Univeristy of Nijmegen.

Berry, J. (2001). A psychology of immigration. Journal of Social Issues, 57(3), 615-631. https://doi.org/10.1111/0022-4537.00231

Berry, J.W. (1997). Immigration, acculturation, and adaptation. Applied Psychology, 46(1), 5-34.

Berry, J.W., Kim, U., Minde, T., \& Mok, D. (1987). Comparative studies of acculturative stress. International Migration Review, pp. 491-511.

Birman, D., \& Trickett, E.J. (2001). Cultural transitions in first-generation immigrants acculturation of soviet jewish refugee adolescents and parents. Journal of Cross-Cultural Psychology, 32(4), 456477.

Boneva, B.S., \& Frieze, I.H. (2001). Toward a concept of a migrant personality. Journal of Social Issues, 57(3), 477-491.

Breidahl, K.N., \& Larsen, C.A. (2016). The Myth of Unadaptable Gender Roles: Attitudes towards Women's Paid Work among Immigrants across 30 European countries. Journal of European Social Policy, 26(5), 387-401. https://doi.org/10.1177/0958928716664292 
Chauvet, L., Gubert, F., \& Mesplé-Somps, S. (2016). Do migrants adopt new political attitudes from abroad? Evidence using a multi-sited exit-poll survey during the 2013 Malian elections.

Comparative Migration Studies, p.4. Retrieved from

https:/comparativemigrationstudies.springeropen.com/articles/10.1186/s40878-016-0033-z

CIA World Factbook. (2011). Retrieved from https://www.cia.gov/library/publications/the-worldfactbook/

Dinesen, P.T. (2012). Does generalized (dis)trust travel? Examining the impact of cultural heritage and destination-country environment on trust of immigrants. Political Psychology, 33(4), 495-511. https://doi.org/10.1111/j.1467-9221.2012.00886.x

Dinesen, P.T. (2013). Where you come from or where you live? Examining the cultural and institutional explanation of generalized trust using migration as a natural experiment. European Sociological Review, 29(1), 114-128.

Dinesen, P.T., \& Hooghe, M. (2010a). When in Rome, do as the romans do: The acculturation of generalized trust among immigrants in western Europe. International Migration Review, 44(3), 697-727.

Dinesen, P.T., \& Hooghe, M. (2010b). When in rome, do as the romans do: The acculturation of generalized trust among immigrants in western Europe. International Migration Review, 44(3), $697-727$.

Eurostat. (2015). Retrieved from http://ec.europa.eu/eurostat/web/population-demography-migrationprojections

Gil, A.G., \& Vega, W.A. (1996). Two different worlds: Acculturation stress and adaptation among Cuban and Nicaraguan families. Journal of Social and Personal Relationships, 13(3), 435-456.

Hedegaard, T.F. (2017). Indvandrergrupper og etniske minoriteter i surveys [Immigrant groups and ethnic minorities in surveys]. In M. Frederiksen, P. Gundelach, \& R.S. Nielsen (Eds.), Survey (p.419). Copenhagen: Hans Reitzels Forlag.

Heleniak, T. (2004). Migration of the russian diaspora after the breakup of the Soviet Union. Journal of International Affairs, 57, 99-117.

Heleniak, T. (2006). Latvia looks west, but legacy of soviets remains. Migration Information Source.

Hirschman, C. (2001). The educational enrollment of immigrant youth: A test of the segmentedassimilation hypothesis. Demography, 38(3), 317-336.

Hox, J.J. (2010). Multilevel analysis: Techniques and applications. Oxford: Taylor \& Francis.

Kahl, S. (2005). The religious roots of modern poverty policy: Catholic, Lutheran, and reformed protestant traditions compared. European Journal of Sociology, 46(1), 91-126. https://doi.org/10.1017/S0003975605000044

Kao, G., \& Tienda, M. (2005). Optimism and achievement: The educational performance of immigrant youth. The New Immigration: An Interdisciplinary Reader, pp. 331-343.

Kesküla, E. (2015). Reverse, restore, repeat! Class, ethnicity, and the Russian-speaking miners of Estonia. Focaal, 2015(72), 95-108.

Kolstø, P. (1996). The new Russian diaspora-an identity of its own? Possible identity trajectories for Russians in the former soviet republic. Ethnic and Racial Studies, 19(3), 609-639.

Lindert, J., von Ehrenstein, O.S., Priebe, S., Mielck, A., \& Brähler, E. (2009). Depression and anxiety in labor migrants and refugees-a systematic review and meta-analysis. Social Science \& Medicine, 69(2), 246-257.

Luttmer, E.F.P. (2001). Group loyalty and the taste for redistribution. Journal of Political Economy, 109(3), 500-528.

Luttmer, E.F.P., \& Singhal, M. (2008). Culture, context, and the taste for redistribution.

Main, I. (2014). High mobility of polish women: The ethnographic inquiry of Barcelona. International Migration, 52(1), 130-145.

Maxwell, R. (2010). Trust in government among British Muslims: The importance of migration status. Political Behavior, 32(1), 89-109. 
Melchior, I., \& Visser, O. (2011). Voicing past and present uncertainties: The relocation of a soviet World War II memorial and the politics of memory in Estonia. Focaal, 2011(59), 33-50.

Nguyen, A.D., \& Benet-Martínez, V. (2013). Biculturalism and adjustment: A meta-analysis. Journal of Cross-Cultural Psychology, 44(1), 122-159.

Nwadiora, E., \& McAdoo, H. (1996). Acculturative stress among Amerasian refugees: Gender and racial differences. Adolescence, 31(122), 477-488.

Ogbu, J.U., \& Simons, H.D. (1998). Voluntary and involuntary minorities: A cultural-ecological theory of school performance with some implications for education. Anthropology \& Education Quarterly, 29(2), 155-188.

Perreira, K.M., Harris, K.M., \& Lee, D. (2006). Making it in America: High school completion by immigrant and native youth. Demography, 43(3), 511-536.

Pfetsch, B. (1999). "In Russia we were Germans, and now we are Russians."-dilemmas of identity formation and communication among German-Russian Aussiedler (Discussion Paper FS III 99103). Retrieved from https://www.econstor.eu/obitstream/10419/49827/1/312915594.pdf

Polek, E., Van Oudenhoven, J.P., \& Berge, J.M.T. (2011). Evidence for a "migrant personality": Attachment styles of poles in Poland and polish immigrants in the Netherlands. Journal of Immigrant \& Refugee Studies, 9(4), 311-326.

Reeskens, T., \& Van Oorschot, W. (2015). Immigrants' attitudes towards welfare redistribution. An exploration of role of government preferences among immigrants and natives across 18 European welfare states. European Sociological Review, 31(4), 433-445.

Röder, A., \& Mühlau, P. (2012). Low expectations or different evaluations: What explains immigrants' high levels of trust in host-country institutions? Journal of Ethnic and Migration Studies, 38(5), 777-792.

Röder, A., \& Mühlau, P. (2014). Are they acculturating? Europe's immigrants and gender egalitarianism. Social Forces, 92(3), 899-928.

Safi, M. (2010). Immigrants' life satisfaction in Europe: Between assimilation and discrimination. European Sociological Review, 26(2), 159-176.

Sodowsky, G.R., Lai, E.W.M., \& Plake, B.S. (1991). Moderating effects of sociocultural variables on acculturation attitudes of Hispanics and Asian Americans. Journal of Counseling \& Development, 70(1), 194-204.

Svallfors, S. (2010). Policy feedback, generational replacement and attitudes to state intervention: Eastern and western Germany, 1990-2006. European Political Science Review, 2(1), 119-135.

Svallfors, S., Kulin, J., \& Schnabel, A. (2012). Age, class, and attitudes towards government responsibilities. In S. Svallfors (Ed.), Contested welfare states. Welfare attitudes in Europe and beyond (1st ed., pp. 158-193).

Tinoco, J.K., \& Ambrose, S.C. (2017). Collaborative Customers and Partners: The Ups and Downs of Their Influence on Firm Technology Competence for New Product Development. Journal of Marketing Development and Competitiveness, 11(4). https://doi.org/10.33423/jmdc.v11i4.1501

Van Hear, N. (2014). Refugees, diasporas and transnationalism. The Oxford Handbook of Refugee and Forced Migration Studies, p.176. 


\section{APPENDIX}

TABLE 1

THE MOBILITY AND VOLUNTARINESS OF CONTACT WITH NATIVES OF DIFFERENT GROUPS

\begin{tabular}{|l|l|l|}
\hline & Voluntary contact & Involuntary contact \\
\hline Sedentary & Ethnic groups & Native Peoples \\
\hline Migrant & Immigrants \& Sojourners & Refugees \\
\hline
\end{tabular}

\section{FIGURE 1 \\ RUSSIAN MIGRANT POPULATIONS IN WESTERN COUNTRIES AND SELECT FORMER SOVIET STATES}

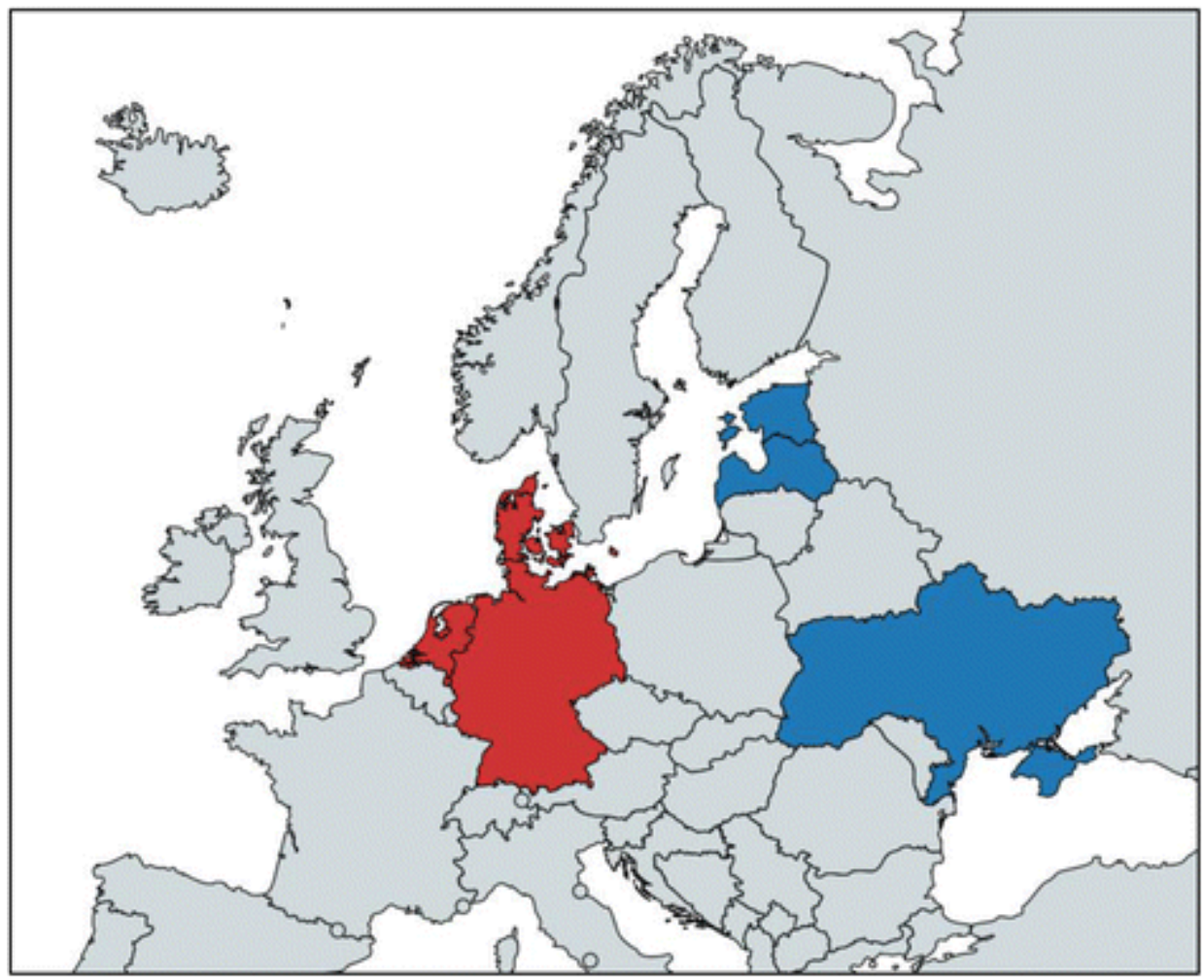

Note: The Western countries highlighted in red are Denmark, Germany and the Netherlands. The countries highlighted in blue are Estonia, Latvia, and Ukraine 
FIGURE 2

MEAN OF DEPENDENT VARIABLES BY GROUP

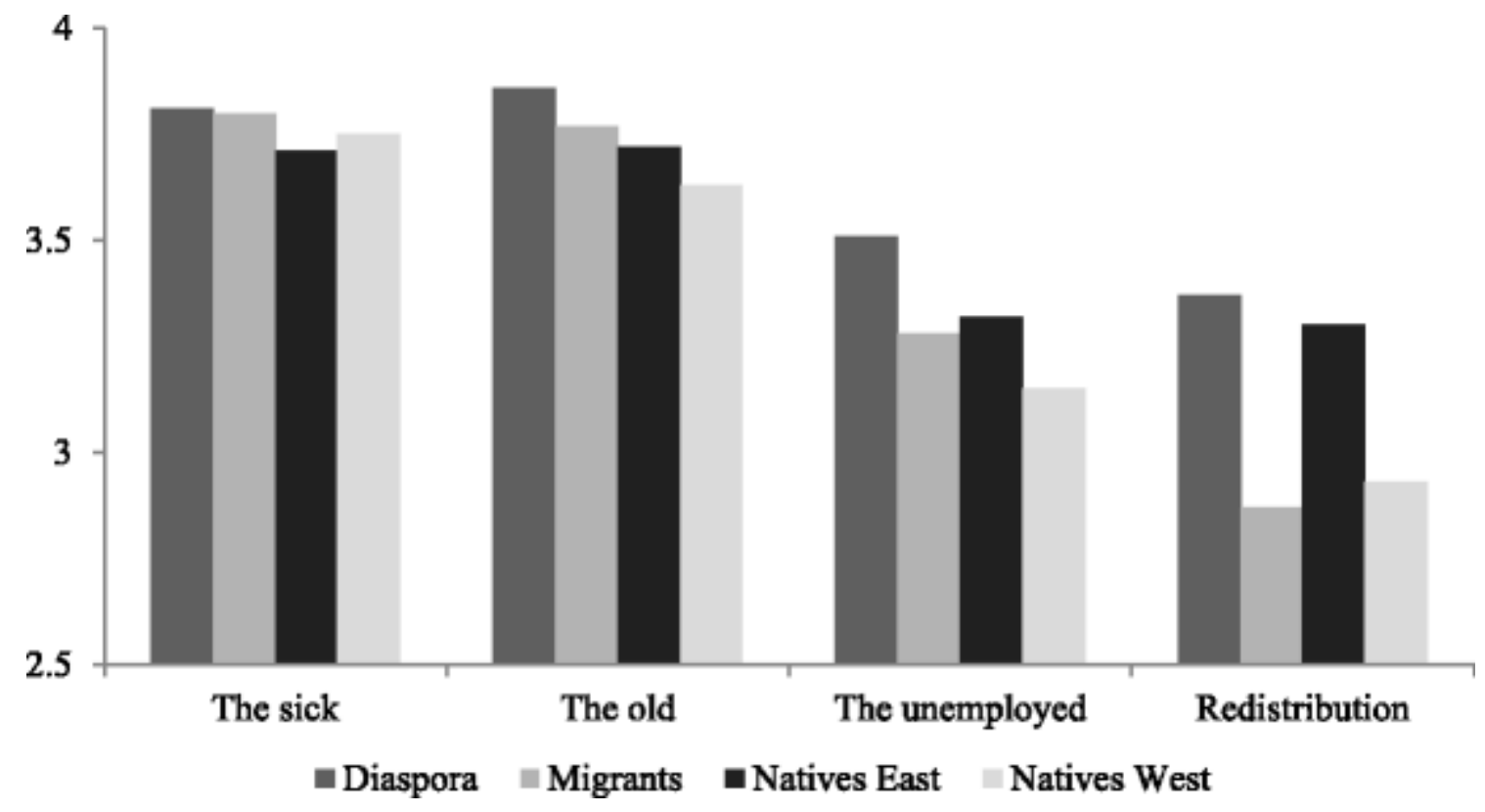

TABLE 2

FIXED EFFECTS REGRESSION MODELS ON ATTITUDES TO GOVERNMENT RESPONSIBILITY. COEFFICIENTS AND SIGNIFICANCE LEVELS REPORTED

\begin{tabular}{|l|c|c|c|c|}
\hline & $\begin{array}{c}\text { Standard of } \\
\text { living for the } \\
\text { sick }\end{array}$ & $\begin{array}{c}\text { Standard of } \\
\text { living for the } \\
\text { old }\end{array}$ & $\begin{array}{c}\text { Standard of living for } \\
\text { the unemployed }\end{array}$ & $\begin{array}{c}\text { Redistribution } \\
\text { between rich and } \\
\text { poor }\end{array}$ \\
\hline $\begin{array}{l}\text { Diaspora } \\
\text { versus } \\
\text { migrants) }\end{array}$ & -0.0288 & -0.0969 & -0.0712 & 0.215 \\
\hline Natives & 0.229 & $0.406^{*}$ & $0.560^{* * *}$ & $0.445^{* *}$ \\
\hline $\begin{array}{l}\text { Age (ref }= \\
>56)\end{array}$ & ref & ref & ref & ref \\
\hline 55-40 & -0.0674 & $-0.0955^{* *}$ & -0.0776 & -0.124 \\
\hline $39-25$ & $-0.126^{* *}$ & -0.0752 & $-0.210^{* *}$ & -0.171 \\
\hline$<25$ & -0.0781 & $-0.165^{* *}$ & $-0.458^{* * *}$ & -0.207 \\
\hline $\begin{array}{l}\text { Age (ref }= \\
>56)\end{array}$ & 0.0457 & 0.0386 & 0.0616 & 0.00658 \\
\hline $\begin{array}{l}\text { Religiosity } \\
(1-4)\end{array}$ & 0.0191 & 0.0265 & 0.0332 & $0.0822^{*}$ \\
\hline
\end{tabular}




\begin{tabular}{|c|c|c|c|c|}
\hline & $\begin{array}{c}\text { Standard of } \\
\text { living for the } \\
\text { sick }\end{array}$ & $\begin{array}{c}\text { Standard of } \\
\text { living for the } \\
\text { old }\end{array}$ & $\begin{array}{c}\text { Standard of living for } \\
\text { the unemployed }\end{array}$ & $\begin{array}{c}\text { Redistribution } \\
\text { between rich and } \\
\text { poor }\end{array}$ \\
\hline $\begin{array}{l}\text { Income } \\
\text { (ref =1 st } \\
\text { quartile) }\end{array}$ & ref & ref & ref & ref \\
\hline 2nd quartile & -0.00386 & -0.0243 & -0.0999 & -0.0785 \\
\hline 3rd quartile & $-0.0937^{* *}$ & -0.0620 & $-0.236^{* * *}$ & $-0.160^{*}$ \\
\hline 4th quartile & -0.0682 & $-0.0854^{*}$ & $-0.308^{* * *}$ & $-0.415^{* * *}$ \\
\hline $\begin{array}{l}\text { Education } \\
\text { (ref= ISCED }\end{array}$ & ref & ref & ref & ref \\
\hline 0) & 0.118 & 0.113 & $0.298^{*}$ & 0.0802 \\
\hline ISCED 1 & 0.0673 & 0.0932 & 0.0541 & 0.152 \\
\hline ISCED 2 & 0.0814 & 0.0836 & 0.154 & -0.117 \\
\hline ISCED 3 & 0.0807 & $0.167^{*}$ & 0.157 & -0.0694 \\
\hline ISCED 4 & 0.0143 & 0.0628 & 0.0348 & -0.119 \\
\hline ISCED 5 & 0.0455 & 0.0820 & -0.0674 & -0.0665 \\
\hline ISCED 6 & 0,00117 & 0,0000482 & $0.0482^{* *}$ & 1285 \\
\hline Social trust \\
(1-5)
\end{tabular}

1. Note: Note: Based on merging ESS and MIFARE data

2. ${ }^{*} p<0.05 ;{ }^{* *} p<0.01 ;{ }^{* * *} p<0.001$

\section{TRANSLATED VERSION: SPANISH}

Below is a rough translation of the insights presented above. This was done to give a general understanding of the ideas presented in the paper. Please excuse any grammatical mistakes and do not hold the original authors responsible for these mistakes.

\section{VERSION TRADUCIDA: ESPAÑOL}

A continuación se muestra una traducción aproximada de las ideas presentadas anteriormente. Esto se hizo para dar una comprensión general de las ideas presentadas en el documento. Por favor, disculpe cualquier error gramatical y no responsabilite a los autores originales de estos errores. 


\section{INTRODUCCIÓN}

Los cambios culturales o institucionales a gran escala son raros. Aunque esto es bueno para la estabilidad de la sociedad, también significa que es difícil probar muchas de las teorías existentes sobre cómo la cultura y las instituciones afectan las actitudes. A excepción de los pocos experimentos naturales, como la reunificación de Alemania Oriental-Occidental (Svallfors, 2010), hay pocas oportunidades para estudiar casos en los que podamos seguir cómo las actitudes se adaptan a un nuevo contexto o cambios culturales. En parte por esta razón, la literatura sobre si las actitudes de los migrantes aculturan ha crecido sustancialmente en los últimos años, ya que, además de proporcionar información sobre la integración de los migrantes en las nuevas sociedades de acogida, estos estudios también ayudan a explorar los mecanismos más generales de formación de actitudes. Los estudios sobre las actitudes de los migrantes incluso se han descrito como "experimentos naturales", ya que muestran lo que sucede cuando un gran grupo de personas con diferentes orígenes entran en contacto con un nuevo país que tiene una cultura diferente y diferentes instituciones. Aunque los resultados de estos estudios son mixtos, la mayoría muestra que los migrantes se aculturan, lo que significa que sus actitudes se parecen más a las de la población nativa que a las dominantes en el país de origen (Breidahl y Larsen, 2016; Chauvet, Gubert y Mesplé-Somps, 2016; Dinesen, 2012; Luttmer \& Singhal, 2008; Maxwell, 2010; Reeskens \& Van Oorschot, 2015; Röder \& Mühlau, 2012; Röder \& Mühlau, 2014; Safi, 2010). Esto indicaría que las actitudes son específicas del contexto y, por lo tanto, cambiantes.

Sin embargo, podría haber un problema con este enfoque de probar los mecanismos de formación de actitudes. Varios estudios, principalmente del campo de la psicología, han argumentado que el acto de migrar podría verse afectado por la voluntad de adaptarse a los valores y actitudes en un nuevo país (Berry, Kim, Minde y Mok, 1987; Berry, 1997, 2001; Birman \& Trickett, 2001; Boneva \& Frieze, 2001; Principal, 2014; Polek, Van Oudenhoven, \& Berge, 2011). Si este es el caso, entonces las conclusiones sobre los mecanismos más generales de formación de actitudes podrían tener que ser reconsideradas. Por lo tanto, queremos demostrar si existe el "efecto de la migración" en la capacidad de aculturación.

Sin embargo, existen una serie de problemas relacionados con la comparación del impacto de la dimensión migratoria en la aculturación, ya que esto a menudo implica comparaciones de grupos culturalmente muy diferentes. Para superar los problemas de la literatura esbozamos un diseño novedoso en el que el colapso de la Unión Soviética sirve como un experimento natural. La caída del Muro de Berlín en 1989 marcó el principio del fin para la Unión Soviética, y unos 2 años más tarde el colapso fue definitivo. Esto significó, entre muchas otras cosas, que los estados de la unión ahora se convirtieron en naciones soberanas nuevamente, lo que resultó en que 25 millones de rusos se convirtieran en "parte de una gran diáspora, sin moverse ni centímetro ni salir de sus hogares" (Heleniak, 2004). Por lo tanto, grandes comunidades de rusos viven en los antiguos estados soviéticos, y estas diásporas constituyen una parte considerable de la población en países como Estonia $(24,8 \%)$, Letonia $(26,2 \%)$ y Ucrania $(17,3 \%)$ (CIA World Factbook, 2011). Al comparar hasta qué punto las actitudes de los países étnicos que viven en Rusia, los países mencionados y los migrantes rusos que viven en Europa Occidental son similares a las de los nativos en sus respectivos países, estudiaremos si existe este "efecto migrante".

Mediremos el nivel de aculturación a las actitudes de los nativos combinando la "Encuesta Social Europea' (ESS) de 2008 con la encuesta 'Actitudes del Estado de Bienestar de los Migrantes' (MIFARE) de 2016, que se recopiló entre diez grupos de migrantes, incluidos los rusos, en los Países Bajos, Dinamarca y Alemania. Usando esta combinación, estudiaremos las actitudes de los rusos desplazados con respecto a si proporcionar atención médica a los enfermos, un nivel de vida garantizado para los ancianos y los desempleados, y la redistribución de recursos debe ser responsabilidad del gobierno.

\section{CONCLUSIÓN}

Una serie de estudios que han salido en los últimos años se centran en las actitudes de los migrantes y si los migrantes aculturan, es decir, si se adaptan para parecerse más a los nativos. Los estudios muestran predominantemente que las actitudes de los migrantes son específicas del contexto y, por lo tanto, 
adaptables (Breidahl y Larsen, 2016; Luttmer \& Singhal, 2008; Maxwell, 2010; Reeskens \& Van Oorschot, 2015; Röder \& Mühlau, 2014; Safi, 2010). Además de informar el debate sobre la integración de los migrantes, estos estudios también se han utilizado para explorar los mecanismos generales de formación de actitudes, ya que la migración se ve como un "experimento natural" en el que podemos observar si el cambio de contexto también puede cambiar las actitudes. Basándonos en teorías, principalmente de la psicología, argumentamos que esta praxis de usar la migración para probar los mecanismos de formación de actitudes podría ser problemática si ser un migrante solo tiene un impacto en la aculturación.

Para explorar esto esbozamos un diseño de estudio basado en un verdadero experimento natural: el colapso de la Unión Soviética. Este evento histórico resultó en una diáspora de más de 25 millones de personas "dejadas atrás" en países que solían pertenecer a la unión. La diáspora rusa se convirtió en una minoría en los nuevos países involuntariamente. Al comparar su aculturación con la de los rusos que emigraron voluntariamente, somos los primeros en probar el "efecto migratorio". En comparación con la literatura existente, este diseño tiene la ventaja de que podemos identificar el efecto de la migración, manteniendo constante el efecto del país de origen y la cultura. Basándonos en la literatura, esbozamos tres escenarios posibles: Primero, que la diáspora acultura con más éxito que los migrantes, como lo predijo la literatura que sigue a Berry et al. (1987). En segundo lugar, que los migrantes rusos se aculturan mejor que la diáspora, como por ejemplo predice la literatura sobre el "optimismo migrante". Tercero, que los migrantes y la diáspora se aculturan a las actitudes de los nativos casi igual de bien. Si no encontramos que este último escenario es el más realista, entonces la investigación actual sobre la adaptación de la formación de actitudes a un nuevo contexto podría haber sobreestimado, o subestimado, el efecto del contexto y las instituciones.

Para probar esto, fusionamos la cuarta versión de la Encuesta Social Europea con la Encuesta de Actitudes del Estado de Bienestar de los Migrantes. Al combinar las dos encuestas, pudimos comparar a los rusos de la diáspora en Estonia, Letonia y Ucrania, y a los migrantes rusos en Dinamarca, los Países Bajos y Alemania, entre sí y con los nativos en los respectivos países. Los resultados mostraron que la diáspora rusa en los dos países bálticos y Ucrania de hecho prefería una responsabilidad más amplia para el gobierno en la prestación de bienestar, lo que podría interpretarse como un apoyo inicial para el efecto migratorio. Sin embargo, los resultados también muestran que esta diferencia se debe principalmente a que la diáspora en promedio es mayor y más pobre. Por lo tanto, al controlar estos factores, no hubo diferencia en la aculturación de las actitudes de los nativos entre los migrantes y la diáspora.

Aunque argumentamos que el "efecto migrante" no existía, existen algunas limitaciones en el diseño de la investigación que deben mejorarse antes de afirmar que no existe una personalidad migrante, independientemente del contexto y el país de origen. El diseño perfecto requeriría que los migrantes y la diáspora estuvieran dentro del mismo contexto. Sin embargo, es difícil imaginar una situación en el mundo real en la que este pueda ser el caso. Con eso en mente, es una limitación que no podamos capturar todas las diferencias contextuales en el estudio. En segundo lugar, solo exploramos esto usando rusos como el caso. No vemos ninguna razón por la que los rusos deban ser más o menos propensos que otras nacionalidades a aculturarse, pero una mayor variación en la nacionalidad, por supuesto, fortalecería la conclusión. En tercer lugar, la diáspora rusa es bastante homogénea en términos de factores como la edad, los ingresos y la educación. Hubiera sido bueno tener más variación para probar el impacto de estos factores; sin embargo, dada la naturaleza del experimento esto no fue posible. Finalmente, está la siempre presente cuestión de la autoselección. Según Heleniak (2004), siempre ha habido un flujo constante de migración entre Rusia y los países limítrofes. Sin embargo, a partir de la década de 1930, se implementaron políticas que facilitaron el aumento de la migración de Rusia a los otros estados de la unión. Esto resultó en un aumento de la migración neta, especialmente a las zonas urbanas, en las que los rusos podían formar comunidades. Aunque la migración inicial fue voluntaria, no se puede descartar que la decisión de migrar antes de la caída de la Unión Soviética y no regresar después, lo que hicieron 2,1 millones de rusos, significa algún nivel de autoselección. De los migrantes que observamos en Europa Occidental, menos del 1\% emigraron antes de la caída de la unión. Por lo tanto, aunque no conocemos razones específicas para migrar, podemos suponer que la acción fue voluntaria y no una huida del dominio soviético. Sin embargo, al igual 
que con aquellos que inicialmente emigraron a los países del Este pero desde el punto de vista opuesto, es imposible saber si existe un elemento de compulsión autopercibida.

A pesar de estas limitaciones, creemos que este estudio es la mejor prueba posible del "efecto migrante". Para el creciente campo de los estudios de migración, esto significa que la migración se puede utilizar para estudiar la formación de actitudes en un sentido más general. Las encuestas sobre migrantes muestran no solo las actitudes de un grupo selecto de personas de un determinado país de origen, sino también las actitudes de las personas de un determinado país de origen que se están adaptando a su nuevo entorno. La pregunta para futuras investigaciones es qué tan rápido irá este proceso de aculturación de actitudes, y qué factores afectan la velocidad del proceso de aculturación.

\section{TRANSLATED VERSION: FRENCH}

Below is a rough translation of the insights presented above. This was done to give a general understanding of the ideas presented in the paper. Please excuse any grammatical mistakes and do not hold the original authors responsible for these mistakes.

\section{VERSION TRADUITE: FRANÇAIS}

Voici une traduction approximative des idées présentées ci-dessus. Cela a été fait pour donner une compréhension générale des idées présentées dans le document. Veuillez excuser toutes les erreurs grammaticales et ne pas tenir les auteurs originaux responsables de ces erreurs.

\section{INTRODUCTION}

Les changements culturels ou institutionnels à grande échelle sont rares. Bien que cela soit bon pour la stabilité de la société, cela signifie également qu'il est difficile de tester de nombreuses théories existantes sur l'impact de la culture et des institutions sur les attitudes. À l'exception des quelques expériences naturelles, comme la réunification est-ouest allemande (Svallfors, 2010), il y a peu d'occasions d'étudier les cas dans lesquels nous pouvons suivre comment les attitudes s'adaptent à un nouveau contexte ou à des changements culturels. C'est en partie pour cette raison que la littérature sur l'acculturation des attitudes des migrants s'est considérablement développée au cours des dernières années car, en plus de donner un aperçu de l'intégration des migrants dans de nouvelles sociétés d'accueil, ces études aident également à explorer les mécanismes plus généraux de formation des attitudes. Les études sur les attitudes des migrants ont, dans certains cas, même été décrites comme des « expériences naturelles », car elles montrent ce qui se passe lorsqu'un grand groupe de personnes d'origines diverses entre en contact avec un nouveau pays ayant une culture et des institutions différentes. Bien que les résultats de ces études soient mitigés, la majorité montre que les migrants s'acculturent, ce qui signifie que leurs attitudes ressemblent davantage à celles de la population autochtone qu'à celles qui dominent dans le pays d'origine (Breidahl et Larsen, 2016; Chauvet, Gubert et Mesplé-Somps, 2016; Dinesen, 2012; Luttmer et Singhal, 2008; Maxwell, 2010; Reeskens et Van Oorschot, 2015; Röder \& Mühlau, 2012 ; Röder \& Mühlau, 2014; Safi, 2010). Cela indiquerait que les attitudes sont spécifiques au contexte et donc modifiables.

Il pourrait cependant y avoir un problème avec cette approche consistant à tester les mécanismes de formation des attitudes. Un certain nombre d'études, principalement dans le domaine de la psychologie, ont soutenu que l'acte de migrer pourrait affecter la volonté de s'adapter aux valeurs et aux attitudes dans un nouveau pays (Berry, Kim, Minde et Mok, 1987; Berry, 1997, 2001; Birman et Trickett, 2001; Boneva et Frieze, 2001; Main, 2014; Polek, Van Oudenhoven et Berge, 2011). Si tel est le cas, alors les conclusions sur les mécanismes plus généraux de formation des attitudes pourraient devoir être reconsidérées. Par conséquent, nous voulons démontrer si « l'effet migratoire » sur la capacité d'acculturation existe.

Il existe cependant un certain nombre de problèmes liés à la comparaison de l'impact de la dimension migratoire sur l'acculturation, car cela implique souvent des comparaisons de groupes culturellement très différents. Pour surmonter les problèmes de la littérature, nous décrivons un nouveau projet dans lequel 
l'effondrement de l'Union soviétique sert d'expérience naturelle. La chute du mur de Berlin en 1989 a marqué le début de la fin pour l'Union soviétique, et environ 2 ans plus tard, l'effondrement était définitif. Cela signifiait, entre autres choses, que les États de l'Union redevenaient des nations souveraines, ce qui a permis à 25 millions de Russes de faire " partie d'une grande diaspora, sans bouger d'un pouce ni quitter leurs foyers » (Heleniak, 2004). De grandes communautés de Russes vivent donc dans les anciens États soviétiques, et ces diasporas constituent une part importante de la population dans des pays comme l'Estonie (24,8\%), la Lettonie (26,2\%) et l'Ukraine (17,3\%) (CIA World Factbook, 2011). En comparant dans quelle mesure les attitudes des Russes ethniques vivant dans les pays susmentionnés et des migrants russes vivant en Europe occidentale sont similaires à celles des natifs de leurs pays respectifs, nous étudierons si cet « effet migrant » existe.

Nous mesurerons le niveau d'acculturation des attitudes des indigènes en combinant l'enquête sociale européenne (ESS) de 2008 avec l'enquête MIFARE (Migrants' Welfare State Attitudes) de 2016, qui a été collectée auprès de dix groupes de migrants, dont des Russes, aux Pays-Bas, au Danemark et en Allemagne. En utilisant cette combinaison, nous étudierons les attitudes des Russes déplacés quant à savoir si la fourniture de soins de santé aux malades, un niveau de vie garanti pour les personnes âgées et les chômeurs, et la redistribution des ressources devraient être la responsabilité du gouvernement.

\section{CONCLUSION}

Un certain nombre d'études qui ont été publiés ces dernières années se concentrent sur les attitudes des migrants et sur la question de savoir si les migrants s'acculturent, c'est-à-dire s'ils s'adaptent pour ressembler davantage aux indigènes. Les études montrent principalement que les attitudes des migrants sont spécifiques au contexte et donc adaptables (Breidahl \& Larsen, 2016; Luttmer et Singhal, 2008; Maxwell, 2010; Reeskens et Van Oorschot, 2015; Röder \& Mühlau, 2014; Safi, 2010). En plus d'éclairer le débat sur l'intégration des migrants, ces études ont également été utilisées pour explorer les mécanismes généraux de formation des attitudes, car la migration est considérée comme une « expérience naturelle » dans laquelle nous pouvons observer si le changement de contexte peut également changer les attitudes. En nous appuyant sur des théories, principalement issues de la psychologie, nous soutenons que cette pratique de l'utilisation de la migration pour tester les mécanismes de formation des attitudes pourrait être problématique si le fait d'être un migrant seul a un impact sur l'acculturation.

Pour explorer cela, nous décrivons un plan d'étude s'appuyant sur une véritable expérience naturelle: l'effondrement de l'Union soviétique. Cet événement historique s'est traduit par une diaspora de plus de 25 millions de personnes " laissées pour compte » dans les pays qui appartenaient à l'Union. La diaspora russe est devenue une minorité dans les nouveaux pays involontairement. En comparant leur acculturation à celle des Russes qui ont émigré volontairement, nous sommes les premiers à tester " l'effet migratoire ». Par rapport à la littérature existante, cette conception a l'avantage de pouvoir identifier l'effet de la migration, tout en maintenant l'effet du pays d'origine et de la culture constant. Sur la base de la littérature, nous avons esquissé trois scénarios possibles: premièrement, que la diaspora acculture avec plus de succès que les migrants, comme prédit par la littérature suivant Berry et al. (1987). Deuxièmement, que les migrants russes s'acculturent mieux que la diaspora, comme le prédisent par exemple la littérature sur « l'optimisme des migrants ». Troisièmement, que les migrants et la diaspora s'acculturent tout autant des attitudes des indigènes. Si nous n'avions pas trouvé ce dernier scénario le plus réaliste, les recherches actuelles sur l'adaptation de la formation des attitudes à un nouveau contexte auraient pu surestimer, ou sous-estimer, l'effet du contexte et des institutions.

Pour tester cela, nous avons fusionné la quatrième version de l'Enquête sociale européenne avec l'enquête sur les attitudes des migrants en matière d'État-providence. En combinant les deux enquêtes, nous avons pu comparer les Russes de la diaspora en Estonie, en Lettonie et en Ukraine, et les migrants russes au Danemark, aux Pays-Bas et en Allemagne, entre eux et avec des natifs dans les pays respectifs. Les résultats ont montré que la diaspora russe dans les deux pays baltes et en Ukraine préférait en fait une responsabilité plus étendue pour le gouvernement dans la fourniture de bien-être, ce qui pourrait être interprété comme un soutien initial à l'effet migratoire. Cependant, les résultats montrent également que 
cette différence est principalement due au fait que la diaspora est en moyenne plus âgée et plus pauvre. Par conséquent, en contrôlant ces facteurs, il n'y avait aucune différence d'acculturation aux attitudes des autochtones entre les migrants et la diaspora.

Bien que nous soutenions que « l'effet migrant » n'existait pas, il existe certaines limites à la conception de la recherche qui devraient être améliorées avant d'affirmer qu'une personnalité migrante, indépendamment du contexte et du pays d'origine, n'existe pas. La conception parfaite exigerait que les migrants et la diaspora soient dans le même contexte. Il est cependant difficile d'imaginer une situation dans le monde réel où cela pourrait être le cas. Dans cet esprit, c'est une limite que nous pourrions ne pas saisir toutes les différences contextuelles dans l'étude. Deuxièmement, nous n'explorons cela qu'en utilisant les Russes comme cas. Nous ne voyons aucune raison pour laquelle les Russes devraient être plus ou moins susceptibles que les autres nationalités de s'acculturer, mais une plus grande variation de nationalité renforcerait bien sûr la conclusion. Troisièmement, la diaspora russe est assez homogène en termes de facteurs tels que l'âge, le revenu et l'éducation. Il aurait été bon d'avoir plus de variations pour tester l'impact de ces facteurs; cependant, compte tenu de la nature de l'expérience, cela n'a pas été possible. Enfin, il y a la question toujours présente de l'auto-sélection. Selon Heleniak (2004), il y a toujours eu un flux constant de migration entre la Russie et les pays limitrophes. Cependant, à partir des années 1930, des politiques ont été mises en œuvre qui ont facilité une migration accrue de la Russie vers les autres États de l'Union. Cela a entraîné une augmentation de la migration nette, en particulier vers les zones urbaines, dans lesquelles les Russes pouvaient former des communautés. Bien que la migration initiale ait été volontaire, il n'est pas exclu que la décision de migrer avant la chute de l'Union soviétique et de ne pas revenir après, ce que 2,1 millions de Russes ont fait, signifie un certain niveau d'autosélection. Parmi les migrants que nous avons examinés en Europe occidentale, moins de 1\% ont migré avant la chute de 1'Union. Par conséquent, bien que nous ne connaissions pas de raisons spécifiques pour migrer, nous pouvons supposer que l'action était volontaire et non une fuite de la domination soviétique. Cependant, comme pour ceux qui ont d'abord migré vers les pays de l'Est, mais du point de vue opposé, il est impossible de savoir s'il existe un élément de compulsion auto-perçue.

Malgré ces limites, nous pensons que cette étude est le meilleur test possible de « l'effet migrant». Pour le domaine croissant des études sur la migration, cela signifie que la migration peut être utilisée pour étudier la formation des attitudes dans un sens plus général. Les enquêtes sur les migrants montrent non seulement les attitudes d'un groupe restreint de personnes d'un certain pays d'origine, mais aussi les attitudes des personnes d'un certain pays d'origine qui s'adaptent à leur nouvel environnement. La question pour d'autres recherches est de savoir à quelle vitesse ce processus d'acculturation des attitudes ira et quels facteurs affectent la vitesse du processus d'acculturation.

\section{TRANSLATED VERSION: GERMAN}

Below is a rough translation of the insights presented above. This was done to give a general understanding of the ideas presented in the paper. Please excuse any grammatical mistakes and do not hold the original authors responsible for these mistakes.

\section{ÜBERSETZTE VERSION: DEUTSCH}

Hier ist eine ungefähre Übersetzung der oben vorgestellten Ideen. Dies wurde getan, um ein allgemeines Verständnis der in dem Dokument vorgestellten Ideen zu vermitteln. Bitte entschuldigen Sie

alle grammatikalischen Fehler und machen Sie die ursprünglichen Autoren nicht für diese Fehler verantwortlich.

\section{EINLEITUNG}

Große kulturelle oder institutionelle Veränderungen sind selten. Obwohl dies gut für die Stabilität der Gesellschaft ist, bedeutet es auch, dass es schwierig ist, viele der bestehenden Theorien darüber zu testen, 
wie Kultur und Institutionen Einstellungen beeinflussen. Abgesehen von den wenigen natürlichen Experimenten, wie der ost-westdeutschen Wiedervereinigung (Svallfors, 2010), gibt es nur wenige Möglichkeiten, Fälle zu untersuchen, in denen wir verfolgen können, wie sich Einstellungen an einen neuen Kontext oder kulturelle Veränderungen anpassen. Teilweise aus diesem Grund ist die Literatur darüber, ob sich die Einstellungen von Migranten akkulturieren, in den letzten Jahren erheblich gewachsen, da diese Studien nicht nur Einblicke in die Integration von Migranten in neue Aufnahmegesellschaften geben, sondern auch dazu beitragen, die allgemeineren Mechanismen der Einstellungsbildung zu erforschen. Studien über die Einstellungen von Migranten wurden in einigen Fällen sogar als "natürliche Experimente" bezeichnet, da sie zeigen, was passiert, wenn eine große Gruppe von Menschen mit unterschiedlichem Hintergrund mit einem neuen Land mit einer anderen Kultur und anderen Institutionen in Kontakt kommt. Obwohl die Ergebnisse dieser Studien gemischt sind, zeigt die Mehrheit, dass Migranten akkulturieren, was bedeutet, dass ihre Einstellungen eher denen der einheimischen Bevölkerung ähneln als denen, die im Herkunftsland dominieren (Breidahl \& Larsen, 2016; Chauvet, Gubert \& Mesplé-Somps, 2016; Dinesen, 2012; Luttmer \& Singhal, 2008; Maxwell, 2010; Reeskens \& Van Oorschot, 2015; Röder \& Mühlau, 2012; Röder \& Mühlau, 2014; Safi, 2010). Dies würde darauf hindeuten, dass Einstellungen kontextspezifisch und damit veränderbar sind.

Es könnte jedoch ein Problem mit diesem Ansatz geben, die Mechanismen der Einstellungsbildung zu testen. Eine Reihe von Studien, hauptsächlich aus dem Bereich der Psychologie, haben argumentiert, dass der Akt der Migration die Bereitschaft beeinflussen könnte, sich an die Werte und Einstellungen in einem neuen Land anzupassen (Berry, Kim, Minde, \& Mok, 1987; Berry, 1997, 2001; Birman \& Trickett, 2001; Boneva \& Frieze, 2001; Main, 2014; Polek, Van Oudenhoven, \& Berge, 2011). Wenn dies der Fall ist, müssen möglicherweise Rückschlüsse auf die allgemeineren Mechanismen der Einstellungsbildung überdacht werden. Daher wollen wir zeigen, ob der "Migrationseffekt" auf die Akkulturationsfähigkeit besteht.

Es gibt jedoch eine Reihe von Problemen im Zusammenhang mit dem Vergleich der Auswirkungen der Migrationsdimension auf die Akkulturation, da dies oft Vergleiche von kulturell sehr unterschiedlichen Gruppen mit sich bringt. Um die Probleme in der Literatur zu überwinden, skizzieren wir einen neuartigen Entwurf, in dem der Zusammenbruch der Sowjetunion als natürliches Experiment dient. Der Fall der Berliner Mauer 1989 signalisierte den Anfang vom Ende für die Sowjetunion, und etwa 2 Jahre später war der Zusammenbruch endgültig. Dies bedeutete unter anderem, dass die Staaten in der Union nun wieder souveräne Nationen wurden, was dazu führte, dass 25 Millionen Russen "Teil einer großen Diaspora wurden, ohne sich einen Zentimeter zu bewegen oder ihre Häuser zu verlassen" (Heleniak, 2004). Große Gemeinschaften von Russen leben daher in ehemaligen Sowjetstaaten, und diese Diasporas machen einen beträchtlichen Teil der Bevölkerung in Ländern wie Estland (24,8\%), Lettland (26,2\%) und der Ukraine $(17,3 \%)$ aus (CIA World Factbook, 2011). Indem wir vergleichen, inwieweit die Einstellungen der ethnischen Russen, die in den oben genannten Ländern leben, und der russischen Migranten, die in Westeuropa leben, denen der Einheimischen in ihren jeweiligen Ländern ähneln, werden wir untersuchen, ob dieser "Migranteneffekt" existiert.

Wir werden den Grad der Akkulturation an die Einstellungen der Einheimischen messen, indem wir den "European Social Survey" (ESS) von 2008 mit der Umfrage "Migrants Welfare State Attitudes" (MIFARE) aus dem Jahr 2016 kombinieren, die unter zehn Migrantengruppen, darunter Russen, in den Niederlanden, Dänemark und Deutschland erhoben wurde. Mit dieser Kombination werden wir die Einstellungen der vertriebenen Russen untersuchen, ob die Gesundheitsversorgung für Kranke, ein garantierter Lebensstandard für Alte und Arbeitslose und die Umverteilung von Ressourcen in der Verantwortung der Regierung liegen sollten.

\section{SCHLUSSFOLGERUNG}

Eine Reihe von Studien, die in den letzten Jahren veröffentlicht wurden, konzentrieren sich auf die Einstellungen von Migranten und darauf, ob Migranten sich akkulturieren, dh ob sie sich anpassen, um den Einheimischen mehr zu werden. Die Studien zeigen überwiegend, dass die Einstellungen von Migranten 
kontextspezifisch und damit anpassungsfähig sind (Breidahl \& Larsen, 2016; Luttmer \& Singhal, 2008; Maxwell, 2010; Reeskens \& Van Oorschot, 2015; Röder \& Mühlau, 2014; Safi, 2010). Neben der Information über die Debatte über die Integration von Migranten wurden diese Studien auch verwendet, um die allgemeinen Mechanismen der Einstellungsbildung zu untersuchen, da Migration als "natürliches Experiment" angesehen wird, in dem wir beobachten können, ob sich ändernde Kontexte auch Einstellungen verändern können. Unter Berufung auf Theorien, hauptsächlich aus der Psychologie, argumentieren wir, dass diese Praxis, Migration zu nutzen, um die Mechanismen der Einstellungsbildung zu testen, problematisch sein könnte, wenn ein Migrant allein einen Einfluss auf die Akkulturation hat.

Um dies zu untersuchen, skizzieren wir ein Studiendesign, das sich auf ein wahres natürliches Experiment stützt: den Zusammenbruch der Sowjetunion. Dieses historische Ereignis führte zu einer Diaspora von mehr als 25 Millionen Menschen, die in Ländern, die früher der Union angehörten, "zurückgelassen" wurden. Die russische Diaspora wurde in den neuen Ländern unfreiwillig zur Minderheit. Indem wir ihre Akkulturation mit der von Russen vergleichen, die freiwillig ausgewandert sind, sind wir die ersten, die den "Migrationseffekt" testen. Im Vergleich zur vorhandenen Literatur hat dieses Design den Vorteil, dass wir den Effekt der Migration genau bestimmen können, während die Wirkung von Herkunftsland und Kultur konstant bleibt. Basierend auf der Literatur skizzierten wir drei mögliche Szenarien: Erstens, dass die Diaspora erfolgreicher akkulturiert als die Migranten, wie die Literatur nach Berry et al. (1987) voraussagt. Zweitens, dass sich die russischen Migranten besser akkulturieren als die Diaspora, wie zum Beispiel die Literatur über den "Migrantenoptimismus" voraussagt. Drittens, dass Migranten und die Diaspora sich ungefähr gleich gut an die Einstellungen der Eingeborenen gewöhnen. Wenn wir das letztere Szenario nicht als das realistischste empfinden würden, dann hätte die aktuelle Forschung zur Anpassung der Einstellungsbildung an einen neuen Kontext die Wirkung von Kontext und Institutionen möglicherweise über- oder unterschätzt.

Um dies zu testen, haben wir die vierte Version des Europäischen Sozialerhebungs survey mit der Migrants ' Welfare State Attitudes survey zusammengeführt. Durch die Kombination der beiden Umfragen konnten wir Diaspora-Russen in Estland, Lettland und der Ukraine sowie russische Migranten in Dänemark, den Niederlanden und Deutschland miteinander und mit Einheimischen in den jeweiligen Ländern vergleichen. Die Ergebnisse zeigten, dass die russische Diaspora in den beiden baltischen Ländern und der Ukraine tatsächlich eine umfassendere Verantwortung der Regierung für die Bereitstellung von Sozialleistungen bevorzugte, was als erste Unterstützung für den Migrationseffekt interpretiert werden könnte. Die Ergebnisse zeigen aber auch, dass dieser Unterschied vor allem darauf zurückzuführen ist, dass die Diaspora im Durchschnitt älter und ärmer ist. Daher gab es bei der Kontrolle dieser Faktoren keinen Unterschied in der Akkulturation an die Einstellungen der Einheimischen zwischen Migranten und der Diaspora.

Obwohl wir argumentieren, dass der "Migrant-Effekt" nicht existierte, gibt es einige Einschränkungen des Forschungsdesigns, die verbessert werden sollten, bevor festgestellt wird, dass eine migrantische Persönlichkeit, unabhängig von Kontext und Herkunftsland, nicht existiert. Das perfekte Design würde erfordern, dass sich die Migranten und die Diaspora im selben Kontext befinden. Es ist jedoch schwer, sich eine Situation in der realen Welt vorzustellen, in der dies der Fall sein könnte. In diesem Sinne ist es eine Einschränkung, dass wir möglicherweise nicht alle kontextuellen Unterschiede in der Studie erfassen. Zweitens untersuchen wir dies nur mit Russen als Fall. Wir sehen keinen Grund, warum Russen mehr oder weniger wahrscheinlich als andere Nationalitäten akkulturieren sollten, aber mehr Unterschiede in der Nationalität würden natürlich die Schlussfolgerung verstärken. Drittens ist die russische Diaspora in Bezug auf Faktoren wie Alter, Einkommen und Bildung ziemlich homogen. Es wäre gut gewesen, mehr Variation zu haben, um die Auswirkungen dieser Faktoren zu testen; Angesichts der Art des Experiments war dies jedoch nicht möglich. Schließlich stellt sich die allgegenwärtige Frage der Selbstselektion. Laut Heleniak (2004) gab es immer einen stetigen Migrationsstrom zwischen Russland und den angrenzenden Ländern. $\mathrm{Ab}$ den 1930er Jahren wurde jedoch eine Politik umgesetzt, die eine verstärkte Migration aus Russland in die anderen Staaten der Union ermöglichte. Dies führte zu einem erhöhten Wanderungssaldo vor allem in städtische Gebiete, in denen die Russen Gemeinschaften bilden konnten. Obwohl die anfängliche Migration freiwillig war, kann nicht ausgeschlossen werden, dass die Entscheidung, vor dem Fall der Sowjetunion zu 
migrieren und danach nicht zurückzukehren, was 2,1 Millionen Russen taten, ein gewisses Maß an Selbstselektion bedeutet. Von den Migranten, die wir in Westeuropa untersucht haben, wanderten weniger als 1\% vor dem Fall der Union aus. Obwohl wir keine spezifischen Gründe für die Migration kennen, können wir davon ausgehen, dass die Aktion freiwillig war und keine Flucht vor der sowjetischen Herrschaft. Wie bei denen, die ursprünglich in die östlichen Länder ausgewandert sind, aber aus dem entgegengesetzten Blickwinkel, ist es unmöglich zu wissen, ob es ein Element des selbst wahrgenommenen Zwanges gibt.

Trotz dieser Einschränkungen glauben wir, dass diese Studie der bestmögliche Test für den "MigrantEffekt" ist. Für das wachsende Feld der Migrationsforschung bedeutet dies, dass Migration genutzt werden kann, um die Einstellungsbildung in einem allgemeineren Sinne zu untersuchen. Migrantenbefragungen zeigen nicht nur die Einstellungen einer ausgewählten Gruppe von Menschen aus einem bestimmten Herkunftsland, sondern auch die Einstellungen von Menschen aus einem bestimmten Herkunftsland, die sich an ihre neue Umgebung anpassen. Die Frage für die weitere Forschung ist, wie schnell dieser Prozess der Akkulturation von Einstellungen ablaufen wird und welche Faktoren die Geschwindigkeit des Akkulturationsprozesses beeinflussen.

\section{TRANSLATED VERSION: PORTUGUESE}

Below is a rough translation of the insights presented above. This was done to give a general understanding of the ideas presented in the paper. Please excuse any grammatical mistakes and do not hold the original authors responsible for these mistakes.

\section{VERSÃO TRADUZIDA: PORTUGUÊS}

Aqui está uma tradução aproximada das ideias acima apresentadas. Isto foi feito para dar uma compreensão geral das ideias apresentadas no documento. Por favor, desculpe todos os erros gramaticais e não responsacule os autores originais responsáveis por estes erros.

\section{INTRODUÇÃO}

Mudanças culturais ou institucionais em larga escala são raras. Embora isso seja bom para a estabilidade da sociedade, também significa que é difícil testar muitas das teorias existentes sobre como a cultura e as instituições impactam as atitudes. Com exceção dos poucos experimentos naturais, como a reunificação da Alemanha Oriental-Ocidental (Svallfors, 2010), há poucas oportunidades de estudar casos em que podemos acompanhar como as atitudes se adaptam a um novo contexto ou mudanças culturais. Em parte por essa razão, a literatura sobre se as atitudes dos migrantes se aculturam tem crescido substancialmente nos últimos anos, pois, além de dar insights sobre a integração dos migrantes em novas sociedades hospedeiras, esses estudos também ajudam a explorar os mecanismos mais gerais de formação de atitude. Estudos de atitudes de migrantes têm, em alguns casos, até sido descritos como "experimentos naturais", pois mostram o que acontece quando um grande grupo de pessoas com diferentes origens entra em contato com um novo país com uma cultura diferente e instituições diferentes. Embora os resultados desses estudos sejam mistos, a maioria mostra que os migrantes aculturam, o que significa que suas atitudes se tornam mais parecidas com as da população nativa do que aquelas dominantes no país de origem (Breidahl \& Larsen, 2016; Chauvet, Gubert, \& Mesplé-Somps, 2016; Dinesen, 2012; Luttmer \& Singhal, 2008; Maxwell, 2010; Reeskens \& Van Oorschot, 2015; Röder \& Mühlau, 2012; Röder \& Mühlau, 2014; Safi, 2010). Isso indicaria que as atitudes são específicas do contexto e, portanto, mutáveis.

Pode haver, no entanto, um problema com essa abordagem de testar os mecanismos de formação de atitude. Uma série de estudos, principalmente do campo da psicologia, têm argumentado que o ato de migrar pode ser afetado pela vontade de se adaptar aos valores e atitudes em um novo país (Berry, Kim, Minde, \& Mok, 1987; Berry, 1997, 2001; Birman \& Trickett, 2001; Boneva \& Frieze, 2001; Principal, 2014; Polek, Van Oudenhoven, \& Berge, 2011). Se este for o caso, então conclusões sobre os mecanismos mais gerais 
de formação de atitude podem ter que ser reconsideradas. Por isso, queremos demonstrar se existe o "efeito migração" sobre a capacidade de aculturação.

Há, no entanto, uma série de problemas relacionados à comparação do impacto da dimensão migratória na aculturação, pois isso muitas vezes implica comparações de grupos culturalmente muito diferentes. Para superar os problemas da literatura, delineamos um novo desenho em que o colapso da União Soviética serve como um experimento natural. A queda do Muro de Berlim em 1989 sinalizou o início do fim para a União Soviética, e cerca de 2 anos depois o colapso foi definitivo. Isso significava, entre muitas outras coisas, que os Estados da União agora se tornaram nações soberanas novamente, o que resultou em 25 milhões de russos se tornando uma "parte de uma grande diáspora, sem se mover um centímetro ou sair de suas casas" (Heleniak, 2004). Grandes comunidades de russos vivem, portanto, em antigos estados soviéticos, e essas diásporas compõem uma parte considerável da população em países como Estônia $(24,8 \%)$, Letônia $(26,2 \%)$ e Ucrânia (17,3\%) (CIA World Factbook, 2011). Comparando-se até que ponto as atitudes dos países étnicos russos e dos migrantes russos que vivem na Europa Ocidental são semelhantes às dos nativos em seus respectivos países, estudaremos se esse "efeito migrante" existe.

Mediremos o nível de aculturação às atitudes dos nativos, combinando a "Pesquisa Social Europeia" (ESS) de 2008 com a pesquisa "Atitudes de Estado de Bem-Estar dos Migrantes" (MIFARE) de 2016, que foi coletada entre dez grupos de migrantes, incluindo russos, nos Países Baixos, Dinamarca e Alemanha. Usando essa combinação, estudaremos atitudes dos russos deslocados sobre a prestação de cuidados de saúde para os doentes, um padrão de vida garantido para os idosos e os desempregados, e a redistribuição de recursos deve ser responsabilidade do governo.

\section{CONCLUSÃO}

Uma série de estudos que surgiram nos últimos anos se concentram nas atitudes dos migrantes e se os migrantes se aculturam, o que significa se eles se adaptam para se tornarem mais parecidos com os nativos. Os estudos mostram predominantemente que as atitudes dos migrantes são específicas do contexto e, portanto, adaptáveis (Breidahl \& Larsen, 2016; Luttmer \& Singhal, 2008; Maxwell, 2010; Reeskens \& Van Oorschot, 2015; Röder \& Mühlau, 2014; Safi, 2010). Além de informar o debate sobre a integração dos migrantes, esses estudos também têm sido utilizados para explorar os mecanismos gerais de formação de atitude, pois a migração é vista como um "experimento natural" no qual podemos observar se a mudança de contexto também pode mudar atitudes. A partir de teorias, principalmente da psicologia, argumentamos que essa práxis de usar a migração para testar os mecanismos de formação de atitude pode ser problemática se ser um migrante sozinho tem um impacto na aculturação.

Para explorar isso, delineamos um desenho de estudo que conta com um verdadeiro experimento natural: o colapso da União Soviética. Este evento histórico resultou em uma diáspora de mais de 25 milhões de pessoas "deixadas para trás" em países que pertenciam à união. A diáspora russa tornou-se uma minoria nos novos países involuntariamente. Comparando sua aculturação com a dos russos que migraram voluntariamente, somos os primeiros a testar o "efeito migração". Em comparação com a literatura existente, esse desenho tem a vantagem de que podemos identificar o efeito da migração, mantendo o efeito do país de origem e da cultura constantes. Com base na literatura, delineamos três cenários possíveis: Primeiro, que a diáspora acultura com mais sucesso do que os migrantes, como previsto pela literatura que se segue Berry et al. (1987). Em segundo lugar, que os migrantes russos aculturam melhor do que a diáspora, como, por exemplo, previsto pela literatura sobre o "otimismo migrante". Terceiro, que os migrantes e a diáspora aculturam as atitudes dos nativos sobre igualmente bem. Se não encontrássemos o último cenário como o mais realista, então a pesquisa atual sobre a adaptação da formação de atitude a um novo contexto poderia ter superestimado, ou subestimado, o efeito do contexto e das instituições.

Para testar isso, fundimos a quarta versão da Pesquisa Social Europeia com a pesquisa Atitudes do Estado de Bem-Estar dos Migrantes. Combinando as duas pesquisas, pudemos comparar os russos da diáspora na Estônia, Letônia e Ucrânia, e migrantes russos na Dinamarca, Holanda e Alemanha, entre si e com os nativos dos respectivos países. Os resultados mostraram que a diáspora russa nos dois países bálticos e na Ucrânia preferiram de fato uma responsabilidade mais extensa para o governo em fornecer bem-estar, 
o que poderia ser interpretado como apoio inicial para o efeito migratório. No entanto, os resultados também mostram que essa diferença se deve, em média, à diáspora, sendo, em média, mais velha e mais pobre. Portanto, ao controlar esses fatores, não houve diferença na aculturação às atitudes dos nativos entre os migrantes e a diáspora.

Embora argumentemos que o "efeito migrante" não existia, existem algumas limitações no desenho da pesquisa que devem ser melhoradas antes de afirmar que a personalidade migrante, independentemente do contexto e do país de origem, não existe. O projeto perfeito exigiria que os migrantes e a diáspora riam no mesmo contexto. É, no entanto, difícil imaginar uma situação no mundo real onde este poderia ser o caso. Com isso em mente, é uma limitação que talvez não capturemos todas as diferenças contextuais no estudo. Segundo, só exploramos isso usando russos como o caso. Não vemos razão para que os russos sejam mais ou menos propensos do que outras nacionalidades a aculturarem, mas uma maior variação na nacionalidade, naturalmente, fortaleceria a conclusão. Em terceiro lugar, a diáspora russa é bastante homogênea em termos de fatores como idade, renda e educação. Teria sido bom ter mais variação para testar o impacto desses fatores; no entanto, dada a natureza do experimento isso não foi possível. Finalmente, há a sempre presente questão da auto-seleção. Segundo Heleniak (2004), sempre houve um fluxo constante de migração entre a Rússia e os países fronteiriços. No entanto, a partir da década de 1930, foram implementadas políticas que facilitaram o aumento da migração da Rússia para os outros Estados da União. Isso resultou em um aumento da migração líquida especialmente para áreas urbanas, nas quais os russos poderiam formar comunidades. Embora a migração inicial tenha sido voluntária, não se pode descartar que a decisão de migrar antes da queda da União Soviética e não voltar depois, o que 2,1 milhões de russos fizeram, significa algum nível de auto-seleção. Dos migrantes que examinamos na Europa Ocidental, menos de $1 \%$ migrou antes da queda da união. Portanto, embora não saibamos razões específicas para migrar, podemos presumir que a ação foi voluntária e não um voo do domínio soviético. No entanto, como aqueles que inicialmente migraram para os países orientais, mas do ponto de vista oposto, é impossível saber se há um elemento de compulsão autopersuem.

Apesar dessas limitações, acreditamos que este estudo é o melhor teste possível do "efeito migrante". Para o crescente campo dos estudos migratórios, isso significa que a migração pode ser usada para estudar a formação de atitude em um sentido mais geral. Pesquisas de migrantes mostram não apenas atitudes de um grupo seleto de pessoas de um determinado país de origem, mas as atitudes de pessoas de um determinado país de origem que estão se adaptando ao seu novo ambiente. A questão para novas pesquisas é a rapidez com que esse processo de aculturação de atitudes irá, e quais fatores afetam a velocidade do processo de aculturação. 